\title{
Evaluation of an in vitro fibre fermentation method using feline faecal inocula: repeatability and reproducibility
}

\author{
Guido Bosch ${ }^{1} *$, Lisa Heesen ${ }^{1}$, Karine de Melo Santos ${ }^{1}$, Wilbert F. Pellikaan ${ }^{1}$, John W. Cone ${ }^{1}$ and \\ Wouter H. Hendriks ${ }^{1,2}$ \\ ${ }^{1}$ Animal Nutrition Group, Wageningen University, PO Box 338, 6700 AH Wageningen, The Netherlands \\ ${ }^{2}$ Faculty of Veterinary Medicine, Utrecht University, PO Box 80.151, 3508 TD Utrecht, The Netherlands
}

(Received 14 November 2016 - Final revision received 3 March 2017 - Accepted 23 March 2017)

Journal of Nutritional Science (2017), vol. 6, e25, page 1 of 4

doi:10.1017/jns.2017.22

Abstract

To gain knowledge on the precision of an in vitro method for characterisation of the fermentability of dietary fibres, this study aimed to evaluate the repeatability and reproducibility of such a method. Substrates used were citrus pectin (CP), fructo-oligosaccharides (FOS), guar gum (GG), sugar beet pulp (SBP) and wheat middlings (WM). Each substrate was incubated with faecal inoculum from five cats with three replicates for each substrate-cat combination. Gas production was measured continuously during the $48 \mathrm{~h}$ incubation and SCFA and organic matter disappearance (only SBP and WM) were determined after incubation. Four consecutive runs were performed. The within-run variability (repeatability) was generally lower for the more simple and pure substrates (CP, FOS, GG) than for the more complex substrates containing mixtures of fibres (SBP, WM). Replicates showed high variability, in particular for SCFA profiles and parameters of gas production kinetics. The between-run CV (reproducibility) for the measured parameters were, in general, below $10 \%$ for CP, FOS and GG and higher values were obtained for SBP and WM. It is concluded that for precise dietary fibre characterisation, the number of replicates should be multiple and adjusted according to the variability of the parameters of interest and the complexity of fibres. The method yielded reproducible results with some variation in absolute values obtained, which may have an impact on the significance level of the differences among substrates.

Key words: Cats: Fibre fermentability: In vitro methodology: Gas production: SCFA

Dietary fibres may affect cats' health, digestive processes and faecal characteristics ${ }^{(1)}$. Such effects depend on the properties of dietary fibres including their potential fermentability by the intestinal microbiota. The fermentability of dietary fibres can be characterised by in vitro methods that simulate intestinal fermentation. The methodology includes incubation of the fibrous substrate of interest with a faecal inoculum from the target animal species. Various considerations for such in vitro methods have been addressed ${ }^{(2)}$ including the required number of faecal donors, which is described in a companion article for cats using similar laboratory procedures and substrates as in the present study ${ }^{(3)}$. As with any analytical method, it is important that the results obtained from an in vitro fermentation method are repeatable and reproducible. The repeatability is defined as the measure of variation for analyses within the same run, whereas reproducibility is the variation among runs $^{(4)}$. Although these types of precision are routinely evaluated for standard laboratory analyses, few studies have specifically examined these for in vitro fermentation methods. This study aimed to evaluate the repeatability and reproducibility of an in vitro method for characterisation of the fermentability of dietary fibres for cats.

Abbreviations: CP, citrus pectin; FOS, fructo-oligosaccharide; GG, guar gum; $R_{\max }$, maximum rate of gas production; SBP, molassed sugar beet pulp; WM, wheat middlings.

* Corresponding author: G. Bosch, email guido.bosch@wur.nl 


\section{Experimental methods}

\section{Substrates}

Dietary fibres or fibre sources were selected based on their use in cat foods, contrasting chemical composition and anticipated fermentation characteristics ${ }^{(5-7)}$. Substrates were citrus pectin (CP; rapidly and highly fermentable, HM Rapid, TIC Gums), fructo-oligosaccharide (FOS; rapidly and highly fermentable; Orafti ${ }^{\circledR}$ IPS, BENEO-Orafti), guar gum (GG; rapidly and highly fermentable, 8/22, TIC Gums), molassed sugar beet pulp (SBP; slowly and highly fermentable; Research Diet Services) and wheat middlings (WM; slowly and moderately fermentable; Research Diet Services).

\section{Animals, housing and care}

A total of five neutered female European shorthair cats ( 3 to 5 years old), with a mean body weight of 3.4 (SD 0.4) $\mathrm{kg}$ were used during this 4 -week study. Cats did not receive any antibiotics for at least 6 months prior to faecal collections. The cats were part of a larger colony where cats are housed in group rooms with inside and outside areas. For the detailed design of the group rooms and the climate and light schedules, see Van Rooijen et al. ${ }^{(8)}$. Cats were fed a nutritionally complete (i.e. meeting the FEDIAF standards) commercial dry extruded diet (Perfect Fit In-Home; Mars Petcare) for at least 4 weeks prior to the first faeces collection. Each cat was fed individually in its own metabolic cage ${ }^{(8)}$ between 08.30 and 09.30 hours (about $45 \%$ of their daily portion), 12.00 and 13.30 hours (about $10 \%$ ) and at 16.30 hours (about $45 \%$ ). The amount of food provided was appropriate to maintain optimal body weight. In the morning and afternoon cats went to their group room and in the evening and night cats stayed in their own cage. Water was always available ad libitum. Litter trays were only present in the metabolic cage and contained nonabsorbent polyethylene litter (Katkor ${ }^{\mathbb{B}}$; Rein Vet Products). The tray and litter were sterilised with $70 \%$ ethanol on the day of faeces collection. The health status of the animals was monitored daily and cats were weighed weekly. The Animal Care and Use Committee of Wageningen University, Wageningen, the Netherlands Animal approved all care and experimental procedures.

\section{Preparation of inoculum and incubation}

Faeces were transferred within $15 \mathrm{~min}$ of defecation to sterile $250 \mathrm{ml}$ plastic bottles prefilled with $\mathrm{CO}_{2}$ and $250 \mathrm{ml}$ of $\mathrm{CO}_{2}$ was immediately added. The bottle with faeces was closed and transported within $5 \mathrm{~min}$ to the analytical laboratory where faeces were processed to inoculum under a constant stream of $\mathrm{CO}_{2}$. Faeces from cats were not pooled but processed for each cat. Attached litter particles were manually removed from faeces and faeces were diluted 1:9 (w/v) in a $39^{\circ}$ $C$ anaerobic sterile physiological saline solution $(9 \mathrm{~g} / 1 \mathrm{NaCl})$. The diluted mixture was homogenised for $60 \mathrm{~s}$ using a handblender and filtered through nylon fabric (pore size $40 \mu \mathrm{m}$, permeability $30 \%$; PA 40/30, Nybolt). The filtrate was mixed with a prewarmed $\left(39^{\circ} \mathrm{C}\right) \mathrm{N}$-containing medium $^{(9)}$ in a $5: 84$ mixture $(\mathrm{v} / \mathrm{v})$ and gently flushed for 5 min with $\mathrm{CO}_{2}$. The resulting medium/ inoculum mixture was dispensed $(89 \mathrm{ml})$ into pre-warmed and $\mathrm{CO}_{2}$-flushed $250 \mathrm{ml}$ serum bottles (Schott) containing $0.5 \mathrm{~g}$ of substrate. Bottles were then placed in the water-bath $\left(39^{\circ} \mathrm{C}\right)$ and attached to a fully automated gas production equipment ${ }^{(10)}$, which recorded gas production for $48 \mathrm{~h}$. This incubation time is longer than the average total tract transit times observed in cats (young adult cats 36 (SD 14) h; senior cats 26 (SD 6) h) ${ }^{(11)}$, with a orocaecal transit time of approximately $5 \mathrm{~h}^{(12)}$. However, $48 \mathrm{~h}$ was estimated to be required for characterising the fermentation kinetics of SBP and WM. After $48 \mathrm{~h}$ of incubation, fermentation liquids were sampled for determination of SCFA (i.e. acetate, propionate, butyrate, iso-butyrate, valerate, iso-valerate) concentrations and for organic matter disappearance (only for SBP and WM). All incubations were done in triplicate. In addition, the study was repeated resulting in four runs performed over 4 weeks (one run/week).

\section{Chemical analyses}

All substrates were chemically characterised as described by Bosch et al. ${ }^{(3)}$. SCFA analyses as well as organic matter disappearance were analysed as described by Bosch et al. ${ }^{(13)}$.

\section{Calculations and data analyses}

Gas (ml) and SCFA (mmol) productions were expressed per $\mathrm{g}$ organic matter (OM). Monophasic models ${ }^{(14)}$ were fitted to the data for cumulative gas production and the maximum rate of gas production $\left(R_{\max }\right.$ in $\mathrm{ml} /(\mathrm{g} O M \mathrm{~h})$ ) and the time at which it occurred $\left(T_{\max }\right.$ in $\left.\mathrm{h}\right)$ were calculated ${ }^{(15)}$. Acetate, propionate and butyrate were expressed as percentage of total SCFA as was the branched-chain proportion (iso-butyrate + isovalerate). Mean and standard deviation values were calculated for all replicates of each substrate-cat combination within the four runs. $\mathrm{CV}_{\mathrm{r}}$ (standard deviation/mean $\times 100$ ) were computed to describe the repeatability of the method. For the evaluation of the reproducibility, the values of replicates for each substrate-cat combination within a run were averaged and then the average for each substrate within that run was computed. The between-run mean and standard deviation values were calculated and used to compute the $\mathrm{CV}_{\mathrm{R}}$ (standard deviation/mean $\times 100)$. For production of SCFA and gas, differences among substrates within each run were tested for significance by ANOVA with Tukey pairwise comparisons.

\section{Results and discussion}

All cats remained healthy throughout the study except for one cat that produced watery diarrhoea in the week of run 2 , which was not used in the study. The substrates contrasted in terms of fermentation parameters measured, as was anticipated (for parameter values, see Bosch et al. ${ }^{(3)}$ ). Of the 285 SCFA analyses performed, six yielded false results. Fitting of the model for gas production was not possible for SBP and WM.

The total SCFA showed highly repeatable results for the more simple and pure substrate (CP, FOS, GG) whereas 
Table 1. Coefficients of variation (\%) within four runs of in vitro fermentation parameters for fibrous substrates using feline faecal inocula*

\begin{tabular}{|c|c|c|c|c|c|c|}
\hline \multirow[b]{2}{*}{ Parameter } & & \multicolumn{5}{|c|}{ Substrate } \\
\hline & & $\mathrm{CP}$ & FOS & GG & SBP & WM \\
\hline \multirow{2}{*}{ Total SCFA } & Median & 3.0 & $2 \cdot 4$ & $2 \cdot 6$ & $5 \cdot 3$ & $14 \cdot 2$ \\
\hline & Maximum & 5.4 & 9.2 & 7.9 & 13.8 & 35.6 \\
\hline \multirow[t]{2}{*}{ Acetate proportion } & Median & 1.5 & 3.6 & 3.5 & $2 \cdot 7$ & $5 \cdot 8$ \\
\hline & Maximum & $19 \cdot 7$ & $26 \cdot 7$ & 24.5 & $26 \cdot 1$ & $30 \cdot 0$ \\
\hline \multirow[t]{2}{*}{ Propionate proportion } & Median & 3.8 & $5 \cdot 7$ & $3 \cdot 2$ & $4 \cdot 2$ & $6 \cdot 0$ \\
\hline & Maximum & $24 \cdot 0$ & $17 \cdot 7$ & $12 \cdot 6$ & 31.9 & $12 \cdot 7$ \\
\hline \multirow[t]{2}{*}{ Butyrate proportion } & Median & $9 \cdot 8$ & 7.7 & $14 \cdot 3$ & 13.5 & $21 \cdot 8$ \\
\hline & Maximum & 48.6 & 43.7 & $48 \cdot 4$ & $42 \cdot 6$ & 79.5 \\
\hline \multirow[t]{2}{*}{ BCP } & Median & $6 \cdot 0$ & 7.4 & 5.7 & 7.4 & 11.8 \\
\hline & Maximum & $59 \cdot 6$ & $48 \cdot 2$ & $46 \cdot 9$ & $55 \cdot 1$ & 52.6 \\
\hline \multirow[t]{2}{*}{ Gas } & Median & 4.4 & 4.4 & 5.4 & 5.7 & $10 \cdot 2$ \\
\hline & Maximum & 11.7 & 14.6 & $13 \cdot 3$ & $15 \cdot 0$ & 24.1 \\
\hline \multirow[t]{2}{*}{$R_{\max }$} & Median & $19 \cdot 4$ & $16 \cdot 4$ & $21 \cdot 0$ & - & - \\
\hline & Maximum & $45 \cdot 2$ & $51 \cdot 2$ & 54.0 & - & - \\
\hline \multirow[t]{2}{*}{$T_{\max }$} & Median & 11.6 & 7.8 & 23.3 & - & - \\
\hline & Maximum & 79.7 & $26 \cdot 8$ & 85.0 & - & - \\
\hline \multirow[t]{2}{*}{ OMD } & Median & - & - & - & $2 \cdot 7$ & $5 \cdot 0$ \\
\hline & Maximum & - & - & - & $26 \cdot 6$ & $15 \cdot 4$ \\
\hline
\end{tabular}

$\mathrm{CP}$, citrus pectin; FOS, fructo-oligosaccharide; GG, guar gum; SBP, molassed sugar beet pulp; WM, wheat middlings; BCP, branched-chain proportion of total SCFA production; $R_{\max }$, maximum rate of gas production; $T_{\max }$, time at which $R_{\max }$ occurred; $\mathrm{OMD}$, organic matter disappearance.

* Indicated CV values (\%) are based on nineteen sets of three replicates per substrate-cat combination in four incubation runs.

SBP showed a slightly higher median $\mathrm{CV}_{\mathrm{r}}$ value, and $\mathrm{CV}_{\mathrm{r}}$ value for WM was higher than $10 \%$ (Table 1). Also the maximum $\mathrm{CV}_{\mathrm{r}}$ values were higher for the more complex substrates (SBP, WM). Acetate and propionate proportion results were highly repeatable for all substrates, but for the butyrate proportion considerably higher median $\mathrm{CV}_{\mathrm{r}}$ values were obtained. The values for branched-chain proportion were intermediate. Considerably high maximum $\mathrm{CV}_{\mathrm{r}}$ values for the proportions of SCFA were found. Outliers in SCFA profiles were not excluded in the present study, as deviating values may represent normal biological variation. For example, the proportions of acetate, propionate and butyrate for $\mathrm{CP}$ in the four runs were, on average, $69 \cdot 1,20.7$ and $5.4 \%$, but in five out of fifty-six fermentation liquids the proportions were $51 \cdot 1,29 \cdot 5$ and $9.0 \%$. Such values were obtained in one of three replicates and were not associated with a specific cat or run. Furthermore, incubation with FOS, GG, SBP and WM resulted also in consistent alternative profiles. These observations may relate to selective growth of specific microbial communities with one of two distinct metabolic behaviours. This hypothesis is in line with the bimodal distribution of specific taxonomic groups found within the human faecal microbiota $^{(16)}$. For precise analysis of SCFA profile of a substrate, it is important to have at least three replicates, although additional study is required to explore these observations in more detail and determine the minimal number of replicates for precise characterisation of dietary fibre fermentability.

The $\mathrm{CV}_{\mathrm{r}}$ values for gas production and organic matter disappearance showed a similar pattern as total SCFA produced and parameters of gas production kinetics showed considerably higher values. These values were higher than those obtained by Van Laar et al. ${ }^{(4)}$. In the latter study, $\mathrm{CV}_{\mathrm{r}}$ values
Table 2. Coefficients of variation (\%) between four runs of in vitro fermentation parameters for fibrous substrates using feline faecal inocula*

\begin{tabular}{lrrrrr}
\hline & \multicolumn{5}{c}{ Substrate } \\
\cline { 2 - 6 } Parameter & CP & FOS & GG & SBP & WM \\
\hline Total SCFA & 4.4 & 2.4 & 5.0 & 7.8 & 8.3 \\
Acetate proportion & 3.8 & 4.7 & 7.0 & 7.6 & 11.8 \\
Propionate proportion & 9.6 & 6.2 & 9.5 & 11.9 & 14.5 \\
Butyrate proportion & 4.9 & 7.7 & 8.4 & 12.5 & 6.8 \\
BCP & 12.0 & 9.2 & 11.4 & 21.8 & 19.3 \\
Gas & 3.3 & 4.3 & 3.6 & 10.4 & 13.3 \\
$R_{\text {max }}$ & 10.5 & 5.9 & 10.7 & - & - \\
$T_{\text {max }}$ & 4.1 & 4.0 & 6.8 & - & - \\
OMD & - & - & - & 5.9 & 1.2 \\
\hline
\end{tabular}

$\mathrm{CP}$, citrus pectin; FOS, fructo-oligosaccharide; GG, guar gum; SBP, molassed sugar beet pulp; WM, wheat middlings; BCP, branched-chain proportion of total SCFA production; $R_{\max }$, maximum rate of gas production; $T_{\max }$, time at which $R_{\max }$ occurred; $\mathrm{OMD}$, organic matter disappearance.

* Indicated CV values (\%) are based on four incubation runs.

were based on duplicate evaluations of four substrates (soyabean meal, wheat grain, grass silage, maize gluten meal) in three to five runs by five laboratories with rumen fluid as the inoculum source. For gas produced after $72 \mathrm{~h}, \mathrm{CV}_{\mathrm{r}}$ values ranged between 1.4 and $10.3 \%$ and for the parameters of gas production kinetics, similar $\mathrm{CV}_{\mathrm{r}}$ values were obtained.

The $C V_{R}$ values were for the measured parameters were below $10 \%$ for the simple and pure substrates (CP, FOS, GG) and higher values were obtained for SBP and WM (Table 2). The organic matter disappearance values for SBP and WM were highly reproducible. The relatively high $\mathrm{CV}_{\mathrm{R}}$ values for $R_{\max }$ were in line with those reported for parameters of fermentation kinetics by Van Laar et al. ${ }^{(4)}$. The $\mathrm{CV}_{\mathrm{R}}$ values for SCFA and gas production were in line with those reported by McBurney \& Thompson ${ }^{(17)}$ who incubated four substrates (oat bran, wheat bran, red kidney bean and GG) with faeces from one healthy human volunteer for $24 \mathrm{~h}$ on three separate non-defined occasions. The $\mathrm{CV}_{\mathrm{R}}$ ranged from 3 to $10 \%$ for SCFA production whereas that for gas production ranged from 1 to $16 \%$. It should be noted that the reproducibility values in the present study relate to a relatively short time period applied between runs and might deviate when longer intervals are applied.

Of the ten substrate comparisons within each run, consistent patterns among the four runs were found. For total SCFA, four comparisons were significant $(P<0.05)$ and two non-significant. For gas production this was, respectively, six and one, acetate proportion five and one, propionate proportion six and one, butyrate proportion zero and seven, and branched-chain proportion four and three. Fermentation parameters for WM were generally different from the other substrates whereas the significance of the differences between the other substrates was more inconsistent.

In conclusion, for precise dietary fibre characterisation, number of replicates should be further explored and adjusted according to the variability of the parameters of interest and the complexity of fibres. The method yielded reproducible results with some variation in obtained absolute values, which may have an impact on the significance level of the differences among substrates. 


\section{Acknowledgements}

We would like to thank D. Nijkamp-Van de Pol and S. Van Laar-Schuppen for assistance during the execution of the in vitro procedures and M. Breuer for SCFA analyses.

This research was funded by Wageningen University.

G. B. contributed to all aspects of this research including research design, data collection, calculations and manuscript drafting. W. F. P., J. W. C. and W. H. H. contributed to research design, data interpretation and manuscript preparation and L. H. and K. M. S. to data collection, calculations, data interpretation and manuscript preparation.

There were no conflicts of interest.

\section{References}

1. Fahey GC Jr, Flickinger AE, Grieshop CM, et al. (2004) The role of dietary fibre in companion animal nutrition. In Dietary Fibre: Bio-Active Carbobydrates for Food and Feed, pp. 295-328 [JW Van der Kamp, N-G Asp, J Miller Jones and G Schaafsma, editors]. Wageningen, The Netherlands: Wageningen Academic Publishers.

2. Coles LT, Moughan PJ \& Darragh AJ (2005) In vitro digestion and fermentation methods, including gas production techniques, as applied to nutritive evaluation of foods in the hindgut of humans and other simple-stomach animals. Anim Feed Sci Tecbnol 123-124, 421-444.

3. Bosch G, Heesen L, de Melo Santos K, et al. (2017) Evaluation of an in vitro fibre fermentation method using feline faecal inocula: inter-individual variation. J Nutr Sci 6, doi:10.1017/jns.2017.21.

4. Van Laar H, Van Straalen WM, Van Gelder AH, et al. (2006) Repeatability and reproducibility of an automated gas production technique. Anim Feed Sci Technol 127, 133-150.

5. Flickinger EA \& Fahey GC Jr (2002) Pet food and feed applications of inulin, oligofructose and other oligosaccharides. $\mathrm{Br} J$ Nutr 87, S297-S300.
6. Karr-Lilienthal LK, Grieshop CM, Smeets-Peeters MJE, et al. (2002) Selected gelling agents in canned dog food affect nutrient digestibilities and fecal characteristics of ileal cannulated dogs. Arch Anim Nutr 56, 141-153.

7. Barry KA, Wojcicki BJ, Bauer LL, et al. (2011) Adaptation of healthy adult cats to select dietary fibers in vivo affects gas and shortchain fatty acid production from fiber fermentation in vitro. J Anim Sci 89, 3163-3169.

8. Van Rooijen C, Bosch G, Butré CI, et al. (2016) Urinary excretion of dietary Maillard reaction products in healthy adult female cats. J Anim Sci 94, 185-195.

9. Williams BA, Bosch MW, Boer $\mathrm{H}$, et al. (2005) An in vitro batch culture method to assess potential fermentability of feed ingredients for monogastric diets. Anim Feed Sci Technol 123-124, 445-462.

10. Cone JW, Van Gelder AH, Visscher GJW, et al. (1996) Influence of rumen fluid and substrate concentration on fermentation kinetics measured with a fully automated time related gas production apparatus. Anim Feed Sci Technol 61, 113-128.

11. Peachey SE, Dawson JM \& Harper EJ (2000) Gastrointestinal transit times in young and old cats. Comp Biochem Physiol A 126, 85-90.

12. Papasouliotis K, Sparkes AH, Gruffydd-Jones TJ, et al. (1998) Use of the breath hydrogen test to assess the effect of age on orocecal transit time and carbohydrate assimilation in cats. Am J Vet Res $\mathbf{5 9}$, 1299-1302.

13. Bosch G, Pellikaan WF, Rutten PGP, et al. (2008) Comparative in vitro fermentation activity in the canine distal gastrointestinal tract and fermentation kinetics of fiber sources. J Anim Sci 86, 29792989.

14. Groot JCJ, Cone JW, Williams BA, et al. (1996) Multiphasic analysis of gas production kinetics for in vitro fermentation of ruminant feeds. Anim Feed Sci Technol 64, 77-89.

15. Bauer E, Williams BA, Voigt C, et al. (2003) Impact of mammalian pretreatment on the fermentability of carbohydrate-rich feedstuffs. J Sci Food Agric 83, 207-214.

16. Lahti L, Salojärvi J, Salonen A, et al. (2014) Tipping elements in the human intestinal ecosystem. Nat Commun 5, 4344.

17. McBurney MI \& Thompson LU (1987) Effect of human faecal donor on in vitro fermentation variables. Br J Nutr 58, 233-243. 